\title{
Localization Performance in Cellular Networks
}

\author{
Javier Schloemann, Harpreet S. Dhillon, and R. Michael Buehrer
}

\begin{abstract}
When the Global Positioning System is unavailable, cellular networks become the dominant vehicle for positioning. However, no tractable approach exists for gaining general insights into localization performance in such networks. Instead, analysis is often done using deterministic network models or with complex system-level simulations, resulting in highly context-specific insights, which do not translate well to random network topologies. In this paper, we motivate and introduce a new approach for analyzing localization performance in cellular networks using tools from point process theory and stochastic geometry. After presenting the model, easy-to-use expressions are derived for the distributions of base station hearability, a metric which is closelyrelated to localization performance, with and without base station coordination.
\end{abstract}

Index Terms-Localization, cellular networks, hearability, stochastic geometry, Poisson point processes.

\section{INTRODUCTION}

$\mathbf{T}$ HE STUDY OF LOCALIZATION in cellular networks has been around for many years [1], [2]. The driving force behind much of the research is a mandate by the Federal Communications Commission requiring cellular network operators to locate those calling 911 to within certain accuracy requirements [3]. Until recently, these requirements included only outdoor location accuracies. Accordingly, the predominant way cellular network operators have met the requirements of the mandate is by relying on the Global Positioning System (GPS). In January of 2015, the FCC expanded its mandate to include a phase-in of indoor positioning requirements, citing that the bulk of emergency calls now originate indoors [4]. While GPS is reliably available under clear sky conditions, where it provides accurate outdoor positioning, its availability suffers greatly in both urban canyons and indoor scenarios. In these situations, the dominant vehicle for positioning is a fallback to cellular network localization. Presently, however, no approaches exist to analyze the fundamentals of localization performance in such networks. In this paper, we present a new analytical model exploiting concepts from point process theory [5] and stochastic geometry [6] that lends tractability to the study of cellular network localization. Using this model, we derive easy-to-use expressions which provide insight into localization performance with and without base station (BS) coordination.

\section{A. Prior Art and Motivation}

In large part, the lack of a tractable analytical model is due to the fact that BS locations are the result of a very complicated optimization problem which is difficult to model and depends

The authors are with the Mobile and Portable Radio Research Group (MPRG), Wireless@ Virginia Tech, Blacksburg, VA, USA. E-Mail: \{javier, hdhillon, buehrer\}@vt.edu. upon various system parameters. Broadly speaking, BSs are deployed in locations so as to maximize coverage and rate for communication purposes, yet the actual placements are further subject to topographical limitations. Moreover, the BS deployments which optimize communication goals may not be sympathetic to localization goals, potentially resulting in highly suboptimal geolocation systems. Consider that in communication systems, it is ideal for a mobile device to receive a strong signal from its serving BS and weak signals from all its neighbors, whereas localization is not possible unless a mobile device receives usable signals from these neighbors, with accuracy generally improving as more neighbors are heard. To underscore the conflicting nature of the two goals, the need to cater to localization demands has a name among cellular system designers, who refer to it as the hearability problem, due to the fact that increasing hearability from neighboring cells for the purposes of positioning is contrary to the principles of cellular system design [7]. Despite this, the localization literature often presents results using specific device and network layouts, which are typically favorable to location estimation (such as by forcing the device to be localized inside the convex hull of its anchors), but do not necessarily represent what may generally be encountered in cellular networks. Even when the device location is not fixed, but the network is, such as when modeling cellular networks using the popular hexagonal grid model [8], analytical results are difficult to come by. Instead, network designers resort to complex system-level simulations, as is clear from the 3GPP standardization process [9]. Unfortunately, simulation approaches do not typically allow general insights to be obtained, such as how localization is impacted by network design parameters. This motivates the need for tractable analytical models that will provide preliminary design insights and will either circumvent the need for simulations completely or limit the ranges of the simulation parameters.

\section{B. Contributions}

In order to provide a tractable analytical model, we turn to point process theory and stochastic geometry to study the geolocation problem. These tools have been applied in wireless contexts before [10]. In fact, both cellular and ad-hoc networks have been studied using tools from stochastic geometry, with ad-hoc networks being the subject of most of the classical works. Recently, however, tools from stochastic geometry have been increasingly applied to the study of cellular networks [11] and emerging heterogeneous networks [12]. Motivated by these advances, we present a new approach catered to the operation of localization systems and provide analytical expressions for BS hearability with and without BS coordination. 


\section{SySTEM MODEL}

\section{A. Spatial base station layout}

The locations of the BSs are modeled using a homogeneous PPP $\Phi \in \mathbb{R}^{2}$ with density $\lambda[6]$. Due to the stationarity of a homogeneous PPP, the device to be localized is assumed to be located at the origin $o$. If the interference is treated as noise at the receiver, the most appropriate metric that captures link quality is the signal-to-interference-plus-noise ratio (SINR). For the link from some BS $x \in \Phi$ to the origin, the SINR can be expressed as:

$$
\operatorname{SINR}_{x}=\frac{P \mathcal{S}_{x}\|x\|^{-\alpha}}{\sum_{\substack{y \in \Phi \\ y \neq x}} P \mathcal{S}_{y}\|y\|^{-\alpha}+\sigma^{2}},
$$

where $P$ is the transmit power, $\mathcal{S}_{z}$ denotes the independent shadowing affecting the signal from BS $z$ to the origin, $\alpha>2$ is the pathloss exponent, and $\sigma^{2}$ is the noise variance. Note that (1) represents the SINR prior to any processing gain, yet as will be evident in the sequel, positioning systems typically have to work at lower target SINRs, thereby necessitating the need for some form of processing gain. In general, the postprocessing SINR will include some multiplicative factor $\gamma$ representing the processing gain, which depends upon system parameters (e.g. integration time) and is assumed to average out the effect of small scale fading. This is the reason why the SINR expression in (1) does not contain a fast fading term, which is consistent with current models for evaluating cellular positioning performance [8].

\section{B. Selection of participating base stations}

As is common in communication system analyses, serving BSs are selected according to the strongest BS association policy, measured using average signal strength, which typically includes long time-scale effects such as shadowing and pathloss. Now, consider that we desire to use a total of $L$ BSs for positioning. Thus, we assume that the $L$ BSs which provide the highest average received power make up the set of participating BSs. Their successful participation, however, is not guaranteed as there is some post-processing SINR threshold $\beta$ (or equivalently, pre-processing SINR threshold $\beta / \gamma$ ) above which the signals from the participating BSs must arrive in order for them to successfully contribute to the localization procedure. In the absence of shadowing, the set of potential BSs simply corresponds to the set of the $L$ nearest BSs. When shadowing is considered and BSs are selected according to average signal strength, the effect of shadowing may be absorbed as a perturbation in the locations of the BSs provided that the fractional moment $\mathbb{E}\left[\mathcal{S}_{z}^{2 / \alpha}\right]<\infty$ [13], [14]. Thus, when this condition is fulfilled and without loss of generality, we define a new equivalent PPP with density $\lambda \mathbb{E}\left[\mathcal{S}_{z}^{2 / \alpha}\right]$. In doing so, we ensure that the strongest BS association policy in the original PPP is equivalent to the nearest BS association policy in the transformed PPP. For notational simplicity, we will continue to represent the transformed PPP by $\Phi$ with density $\lambda$, under the assumption that if shadowing is present, it

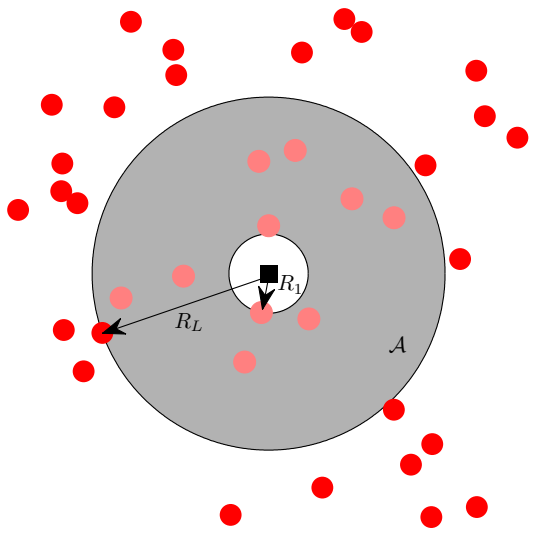

Fig. 1. The PROPOSED MODEL. The mobile device is denoted by the central square and BSs are denoted by the circles. The BSs participating in the localization procedure (within the gray annular region $\mathcal{A}$ ) are either all on or all off during the $L^{\text {th }}$ BS's transmission, depending on the coordination.

is already reflected in the density of $\Phi$. Note that the condition on the fractional moment is fairly mild and will almost always be satisfied by the distributions of interest, including the most common assumption of log-normal shadowing with finite mean and standard deviation [14]. As a final note, it has been shown that for SINR, shadowing causes even more regular network models, such as the common hexagonal lattice, to behave like a PPP model [15], [16]. This further validates the use of a PPP to model BS locations.

\section{Base station coordination}

In cellular networks, backhaul connections allow for the coordination of transmissions between BSs during localization procedures. An example of this technique for improving the hearability of far away BSs is that of joint scheduling in LTE [7]. For simplicity of exposition, let us order the BSs in the now shadowing-transformed $\Phi$ in terms of increasing distance from the origin such that the location of the $k^{\text {th }}$ farthest BS from the origin is denoted by $x_{k} \in \Phi$. We now enrich the previous SINR expression in (1) for the signals arriving from the participating BSs (i.e., $x_{k}$ for $k \in\{1, \ldots, L\}$ ) by including BS coordination:

$$
\operatorname{SINR}_{k}(L)=\frac{P\left\|x_{k}\right\|^{-\alpha}}{\mathbb{1}_{\mathrm{NC}} \cdot \sum_{\substack{i=1 \\ i \neq k}}^{L} P\left\|x_{i}\right\|^{-\alpha}+\sum_{j=L+1}^{\infty} P\left\|x_{j}\right\|^{-\alpha}+\sigma^{2}},
$$

where $\mathbb{1}_{\mathrm{NC}}$ is an indicator taking value unity when there is no coordination between BSs. Note that $\mathrm{SINR}_{k}$ is a function of $L$ because of the potential coordination among the $L$ participating BSs.

\section{Base station range distributions}

From (2), it is clear the SINRs are dependent on the distances of the BSs from the origin, rather than their exact locations. Thus, it is worthwhile to characterize these. Denote the distance of the $k^{\text {th }} \mathrm{BS}$ from the origin by $R_{k}=\left\|x_{k}\right\|$ as illustrated in 
Figure 1 for the closest and $L$ th closest BSs. The distribution of $R_{L}$ is known to be [17]:

$$
f_{R_{L}}(r)=e^{-\lambda \pi r^{2}} \frac{2\left(\lambda \pi r^{2}\right)^{L}}{r(L-1) !} .
$$

Now, conditioned on the location (or distance) of the $L^{\text {th }}$ BS, we present a useful lemma for understanding the distribution of the $L-1$ closest BSs.

Lemma 1. Conditioned on the location of the $L^{\text {th }} B S$, the $L-1 B S s$ closer to the origin are distributed according to a binomial point process (BPP) (i.e., in a uniformly random manner) inside the circle of radius $R_{L}$ centered at the origin.

\section{Proof. See Appendix A.}

Using Lemma 1, we arrive at the following distribution for the most dominant (i.e., closest) interferer, given the distance of the $L^{\text {th }}$ BS. This is formally presented below.

Lemma 2. The cumulative distribution function of the closest $B S$ distance $R_{1}$ given $R_{L}$ is

$$
F_{R_{1} \mid R_{L}}\left(r \mid R_{L}\right)=1-\left(\frac{R_{L}^{2}-r^{2}}{R_{L}^{2}}\right)^{L-1} .
$$

Proof. See Appendix B.

Let $\mathcal{A}=\mathfrak{b}\left(o, R_{L}\right) \backslash \mathfrak{b}\left(o, R_{1}\right)$, where $\mathfrak{b}(\boldsymbol{\theta}, r)$ represents a ball of radius $r$ centered at $\boldsymbol{\theta}$. We have the following lemma characterizing the distribution of the $L-2$ remaining participating BSs in this annular region shown in Figure 1.

Lemma 3. Conditioned on $R_{1}$ and $R_{L}$, the $L-2 B S$ s located inside the annular region $\mathrm{b}\left(o, R_{L}\right) \backslash \mathrm{b}\left(o, R_{1}\right)$ are distributed according to a BPP.

Proof. See Appendix A.

\section{Localization Performance}

Regardless of the technique, cellular geolocation performance fundamentally depends upon three things: (i) the geometry of the participating BSs relative to the device being localized, (ii) the number of participating BSs, and (iii) the accuracy of the positioning observations. These three factors are all highly interdependent. For instance, both the number of BSs whose signals arrive with sufficiently-high SINRs to participate in the localization procedure and the quality of their positioning observations are strongly dependent on the interference field, which is itself driven by the realized network geometry. Thus, a full characterization of localization performance would take into account all possible geometric conditionings of the BSs, a task which is extremely difficult. However, by taking into account even just the number of participating BSs, valuable insights into localization performance may be gleaned. In the present work, we focus on the case of $\alpha=4$ in interference-limited networks, which is commonly of interest in the cellular communication literature. Note that using $\alpha=4$ allows us to obtain simplified analytical expressions, while remaining close to the pathloss exponent of 3.76 accepted for 3GPP simulations [8]. For more general pathloss exponents, the reader is referred to [18].

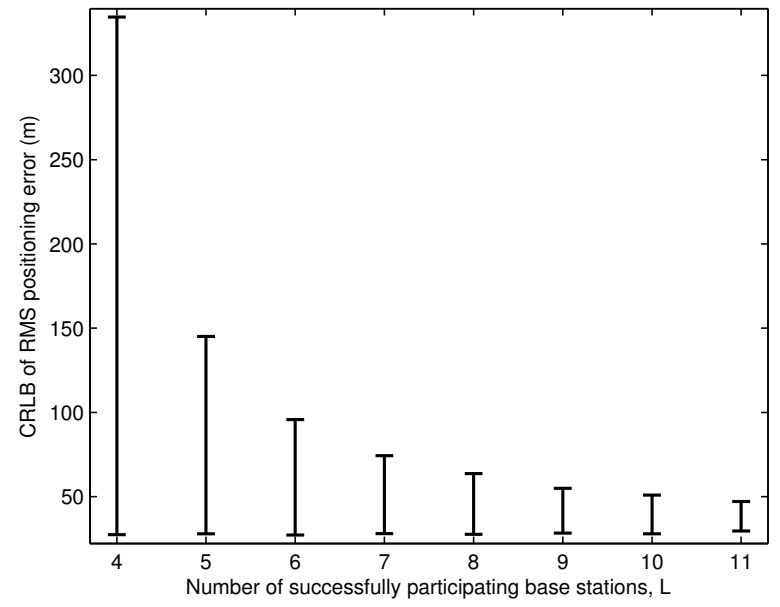

Fig. 2. RELATIONSHIP BETWEEN LOCALIZATION ACCURACY AND BS PARTICIPATION: Ranges of the lower $95^{\text {th }}$ percentiles of positioning accuracies using TDOA positioning. (No BS coordination, $\alpha=4, \beta / \gamma=-16 \mathrm{~dB}$, and $8 \mathrm{~dB}$ shadowing standard deviation.)

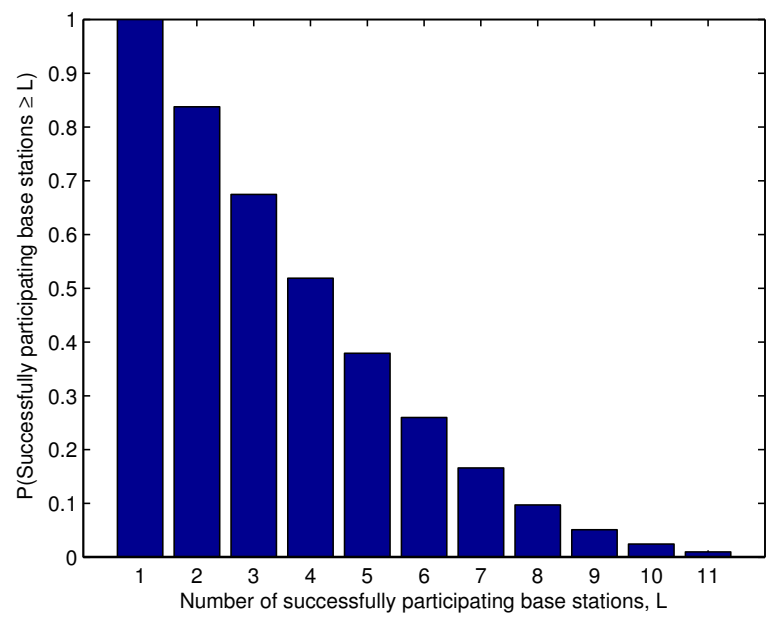

Fig. 3. DeCreasing AVAilability of BSs: A histogram illustrating the decreasing probability of attaining an increasing number of successful BS connections. (The setup is identical to that of Figure 2.)

\section{A. Base station participation and geolocation performance}

A clear relationship exists between the number of BSs involved in positioning and the distribution of the resulting localization accuracy. In order to see this, consider the CramérRao lower bound (CRLB), which provides a lower bound on the achievable performance of an unbiased estimator, applied to time-difference-of-arrival (TDOA) positioning, commonly employed in cellular localization (e.g., observed time-differenceof-arrival, OTDOA, in LTE). Using the model described in the previous section with a BS deployment density equivalent to that of an infinite hexagonal grid with $500 \mathrm{~m}$ intersite distances and a shadowing standard deviation of $8 \mathrm{~dB}$, a localization system without BS coordination was simulated requiring $\beta=-6 \mathrm{~dB}$ for successful signal detection after a processing gain of $\gamma=10 \mathrm{~dB}$. The threshold $\beta$ determines which BSs may successfully participate in the localization procedure, while the BSs' exact SINRs determine the accuracies 
of their individual ranging observations, calculated using the well-known TOA ranging CRLB [19] with an assumed signal bandwidth of $2 \mathrm{MHz}$. Lastly, as is typical, the strongest BS is selected as the reference BS for TDOA. From Figure 2, which plots the range of the lower $95^{\text {th }}$ percentiles of the achievable root-mean-square (RMS) positioning errors observed in the simulation versus the number of successfully participating BSs, we see that not only does the achievable localization performance generally improve with an increasing number of BSs, it starts to become almost predictable. Motivated by this trend, we now formally propose a metric to study the number of BSs able to successfully participate in a localization procedure for a given target SINR $\beta$ and processing gain $\gamma$.

Definition 1 (Participation metric). For a given BS deployment $\phi \in \Phi$, let $\Upsilon$ represent the maximum number of BSs capable of successfully participating in a localization procedure. This can be mathematically defined as:

$$
\Upsilon=\underset{\ell}{\arg \max } \ell \cdot \prod_{k=1}^{\ell} \mathbb{1}\left(\operatorname{SINR}_{k}(\ell) \geq \frac{\beta}{\gamma}\right) .
$$

Note that this metric differs from a traditional metric such as the CRLB in that it is not directly tied to a specific positioning accuracy value since it does not take into account geometry, though the above analysis shows that it is certainly related to performance. Its advantage lies in its tractability for random geometries, whereas the CRLB is easily derived for deterministic geometries, but quickly becomes intractable to characterize for random network geometries.

\section{B. Participation from a desired number of base stations}

Figure 3 plots the probabilities of attaining some desired number of successful BS connections as observed through the simulation. While generally better localization performance can be attained by increasing BS participation, it is clear from Figure 3 that the probability of successfully involving more BSs decreases sharply as the number of BSs increases. Thus, it is desirable to understand exactly how the participation metric $\Upsilon$ is impacted by network design parameters.

Definition 2 ( $L$-localizability probability). For a given $\phi \in \Phi$, a mobile device is said to be L-localizable if at least L BSs may successfully participate in the localization procedure. The probability of this occurring is simply:

$$
\mathrm{P}_{\mathrm{L}}=\mathbb{P}(\Upsilon \geq L) \text {. }
$$

Note that for $L=1$, this simply gives the downlink coverage probability.

When the small scale fading is averaged out, as is the case in the proposed model described in Section II, it is straightforward to infer from (2) that $\mathbb{1}\left(\operatorname{SINR}_{k}(L) \geq \beta\right) \geq \mathbb{1}\left(\operatorname{SINR}_{l}(L) \geq \beta\right)$ for all $k \leq l \leq L$. This simply means that the received SINR from a BS farther from the mobile device is lower than that of a closer BS, meaning that the probability of $L$-localizability can be equivalently expressed as

$$
\mathrm{P}_{\mathrm{L}}=\mathbb{E}_{\Phi}\left[\mathbb{1}\left(\operatorname{SINR}_{L}(L) \geq \beta\right)\right] .
$$

\section{Probability of L-localizability}

For the signal arriving from the $L^{\text {th }}$ participating BS $(L>1)$, let $\mathcal{I}_{1}=\sum_{i=2}^{L-1} P\left\|x_{i}\right\|^{-\alpha}$ be the (potential) interference from the other participating BSs excluding the closest BS (for reasons which will be evident later) and $\mathcal{I}_{2}=\sum_{j=L+1}^{\infty} P\left\|x_{j}\right\|^{-\alpha}$ be the interference from BSs beyond the $L^{\text {th }}$ one. We now consider an approximation of $\mathrm{P}_{\mathrm{L}}$ when $\mathbb{1}_{\mathrm{NC}}=1$, i.e., without BS coordination, by considering the means of $\mathcal{I}_{1}$ and $\mathcal{I}_{2}$ conditioned on $R_{1}$ and $R_{L}$, rather than $\mathcal{I}_{1}$ and $\mathcal{I}_{2}$ themselves. These are characterized in the following lemmas.

Lemma 4. The expected value of $\mathcal{I}_{1}$ conditioned on $R_{1}$ and $R_{L}$ is

$$
\mathbb{E}\left[\mathcal{I}_{1} \mid R_{1}, R_{L}\right]=\frac{2 P(L-2)}{2-\alpha} \cdot \frac{R_{L}^{2-\alpha}-R_{1}^{2-\alpha}}{R_{L}^{2}-R_{1}^{2}} .
$$

Proof. See Appendix C.

Lemma 5. The expected value of $\mathcal{I}_{2}$ conditioned on $R_{L}$ is

$$
\mathbb{E}\left[\mathcal{I}_{2} \mid R_{L}\right]=\frac{2 P \pi \lambda}{\alpha-2} R_{L}^{2-\alpha}
$$

for $\alpha>2$, and unbounded otherwise.

Proof. See Appendix D.

Since cellular networks are typically limited by interference rather than thermal noise, we remove the thermal noise term, and apply Lemmas 4 and 5 to obtain the following approximation for $\mathrm{P}_{\mathrm{L}}$.

Theorem 1 (No base station coordination). When all BSs transmit simultaneously, the probability of L-localizability may be approximated as

$$
\begin{aligned}
& \mathrm{P}_{\mathrm{L}}(L, \beta, \gamma, \lambda)=\int_{0}^{\sqrt{\frac{\gamma / \beta-(L-1)}{\pi \lambda}}}(1- \\
& \left.\frac{1}{\sqrt{\gamma / \beta-\pi \lambda r^{2}+\frac{(L-2)^{2}}{4}}-\frac{L-2}{2}}\right)^{L-1} e^{-\lambda \pi r^{2}} \frac{2\left(\lambda \pi r^{2}\right)^{L}}{r(L-1) !} \mathrm{d} r .
\end{aligned}
$$

Proof. See Appendix E.

Next, we consider the cellular localization scenario when $\mathbb{1}_{\mathrm{NC}}=0$, i.e., with perfect $\mathrm{BS}$ coordination, by again considering the conditional mean of $\mathcal{I}_{2}$. In this case, Lemma 5 may be employed to obtain a simple approximation of $\mathrm{P}_{\mathrm{L}}$.

Theorem 2 (Perfect base station coordination). When all BSs transmit sequentially and remain quiet during the other BSs, transmissions, $\mathrm{P}_{\mathrm{L}}$ may be approximated as

$$
\mathrm{P}_{\mathrm{L}}(L, \beta, \gamma, \lambda)=1-\sum_{\ell=0}^{L-1} e^{-\frac{\gamma}{\beta}} \frac{\left(\frac{\gamma}{\beta}\right)^{\ell}}{\ell !} .
$$

Proof. Following a similar derivation to (10), but excluding the interference from the other $L-1$ participating BSs,

$$
\mathrm{P}_{\mathrm{L}}(L, \beta, \gamma, \lambda)=\int_{0}^{\sqrt{\frac{\gamma / \beta}{\pi \lambda}}} e^{-\lambda \pi r^{2}} \frac{2\left(\lambda \pi r^{2}\right)^{L}}{r(L-1) !} \mathrm{d} r
$$




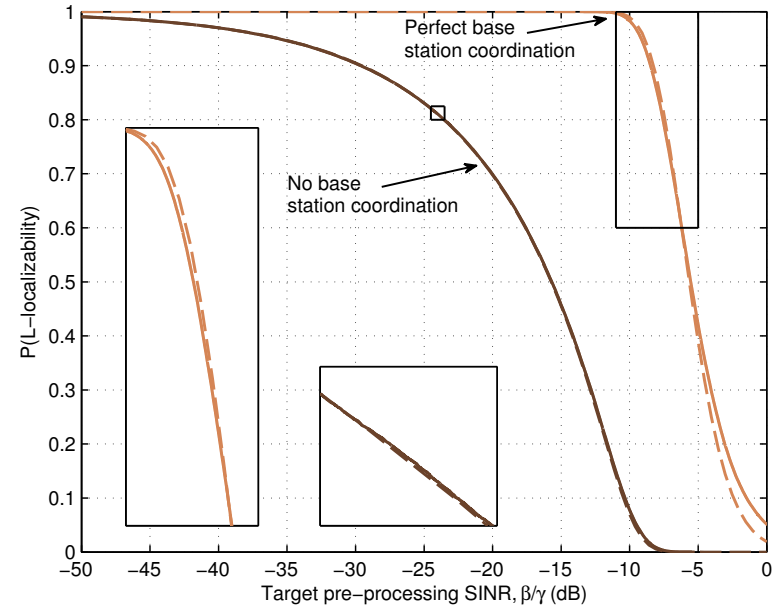

Fig. 4. ACCURACY OF THE APPROXIMATION: This figure illustrates both the true localizability probabilities (solid line) as well as those provided by the approximation (dashed line) for $\mathrm{L}=4$. Note that the approximation is nearly indistinguishable from truth and requires only a simple analytical evaluation.

which is simply the probability of finding at least $L$ nodes inside $\mathfrak{b}\left(o, \sqrt{\frac{\gamma / \beta}{\pi \lambda}}\right)$.

One interesting remark here is that with perfect BS coordination, the density of the BS deployment does not appear to affect $P_{L}$.

\section{NUMERICAL VERIFICATION}

We now verify the accuracy of the approximations by comparing with the true $P_{L}$ gathered using 10,000 random network realizations. In Figure 4, both approximations are plotted for $L=4$, the minimum number of BSs required for unambiguous TDOA positioning. We observe that the approximations are remarkably tight, being nearly indistinguishable from truth except in the low-reliability regime $\left(\mathrm{P}_{\mathrm{L}}<0.4\right)$, which is not very desirable for system design anyway. A key factor enabling these tight approximations is that the locations of the strongest BS (i.e., the dominant interferer) and the $L^{\text {th }}$ BS are considered exactly. To appreciate the value of the tight approximations, consider the exact expression for the $k$-coverage problem in the absence of fast fading in [20, Corr. 7], which applies here to $\mathrm{P}_{\mathrm{L}}$ with no $\mathrm{BS}$ coordination. A cursory glance at the expression reveals its cumbersome nature, involving many multi-fold integrals, from which it is extremely difficult to gain general insights.

\section{CONCLUSION}

We have introduced concepts from point process theory and stochastic geometry to increase the tractability of localization performance analyses in random networks, which may encompass cellular networks as well as WiFi access point deployments. This is in contrast to most previous approaches, which are either specific to deterministic deployments or rely on time-consuming simulations. Furthermore, the value of the model was demonstrated through tight approximations of network hearability which make evident the effects of individual network parameters on localization performance.

\section{APPENDIX}

\section{A. Proof of Lemmas 1 and 3}

Let $i<j, n \leq \Xi, \Xi$ be the number of BSs between $x_{i}$ and $x_{j}$, $A=\mathfrak{b}\left(o,\left\|x_{i}\right\|+d a / 2\right) \backslash \mathfrak{b}\left(o,\left\|x_{i}\right\|-d a / 2\right), B=\mathfrak{b}\left(o,\left\|x_{j}\right\|+\right.$ $d b / 2) \backslash \mathfrak{b}\left(o,\left\|x_{j}\right\|-d b / 2\right), C=\mathfrak{b}\left(o,\left\|x_{j}\right\|-d b / 2\right) \backslash \mathfrak{b}\left(o,\left\|x_{i}\right\|+\right.$ $d a / 2), D \subseteq C, \mathfrak{b}(\boldsymbol{\theta}, r)$ represent a ball of radius $r$ centered at $\boldsymbol{\theta}$, and $N_{\mathcal{H}}$ be the number of points in region $\mathcal{H}$. Then,

$$
\begin{aligned}
& \mathbb{P}\left(N_{D}=n \mid x_{i}, x_{j}\right) \\
& =\lim _{d a, d b \rightarrow 0} \mathbb{P}\left(N_{D}=n \mid N_{C}=\Xi, N_{B}=1, N_{A}=1\right) \\
& =\lim _{d a, d b \rightarrow 0} \frac{\mathbb{P}\left(N_{D}=n, N_{C}=\Xi, N_{B}=1, N_{A}=1\right)}{\mathbb{P}\left(N_{C}=\Xi, N_{B}=1, N_{A}=1\right)} \\
& =\lim _{d a, d b \rightarrow 0} \frac{\mathbb{P}\left(N_{D}=n, N_{C \backslash D}=\Xi-n, N_{B}=1, N_{A}=1\right)}{\mathbb{P}\left(N_{C}=\Xi, N_{B}=1, N_{A}=1\right)} \\
& =\frac{\mathbb{P}\left(N_{D}=n\right) \mathbb{P}\left(N_{C \backslash D}=\Xi-n\right)}{\mathbb{P}\left(N_{C}=\Xi\right)} \\
& =\frac{(\lambda|D|)^{n} e^{-\lambda|D|} / n !}{(\lambda|C|)^{\Xi} e^{-\lambda|C|} \cdot \Xi !} \frac{(\lambda(|C|-|D|))^{\Xi-n} e^{-\lambda(|C|-|D|)}}{(\Xi-n) !} \\
& =\frac{\Xi !}{n !(\Xi-n) !}\left(\frac{|D|}{|C|}\right)^{n}\left(1-\frac{|D|}{|C|}\right)^{\Xi-n}
\end{aligned}
$$

which shows that the $\Xi$ BSs inside the annular region $\lim _{d a, d b \rightarrow 0} C=\mathfrak{b}\left(o,\left\|x_{j}\right\|\right) \backslash \mathfrak{b}\left(o,\left\|x_{i}\right\|\right)$ make up a BPP. By letting $i=0, j=L$, and defining $x_{0} \triangleq o$ (i.e., $\left\|x_{0}\right\|=0$ ), the $\mathrm{BSs}$ inside the circular region $\mathfrak{b}\left(o,\left\|x_{L}\right\|\right)$ are shown to make up a BPP, thus proving Lemma 1 . By letting $i=1$ and $j=L$, Lemma 3 is proved.

B. Proof of Lemma 2

$$
\begin{aligned}
& F_{R_{1} \mid R_{L}}\left(r \mid R_{L}\right)=\mathbb{P}\left(R_{1} \leq r \mid R_{L}\right)=1-\mathbb{P}\left(R_{1}>r \mid R_{L}\right) \\
& =1-\mathbb{P}\left(\min \left(\left\{x:\|x\|<\left\|x_{L}\right\|\right\}\right)>r \mid R_{L}\right) \\
& \stackrel{(a)}{=} 1-\prod_{\left\{x:\|x\|<\left\|x_{L}\right\|\right\}} \mathbb{P}\left(\|x\|>r \mid R_{L}\right) \\
& =1-\prod_{\left\{x:\|x\|<\left\|x_{L}\right\|\right\}} \frac{\pi R_{L}^{2}-\pi r^{2}}{\pi R_{L}^{2}}=1-\left(\frac{R_{L}^{2}-r^{2}}{R_{L}^{2}}\right)^{L-1},
\end{aligned}
$$

where (a) follows from Lemma 3.

\section{Proof of Lemma 4}

Let $x$ be one of the BSs between $x_{1}$ and $x_{L}$ in distance from the origin. Then

$$
\begin{aligned}
& \mathbb{E}\left[P\|x\|^{-\alpha} \mid R_{1}, R_{L}\right] \stackrel{(a)}{=} \int_{R_{1}}^{R_{L}} \operatorname{Pr}^{-\alpha} \frac{2 r}{R_{L}^{2}-R_{1}^{2}} \mathrm{~d} r \\
& =\frac{2 P}{R_{L}^{2}-R_{1}^{2}} \int_{R_{1}}^{R_{L}} r^{1-\alpha} \mathrm{d} r=\frac{2 P}{R_{L}^{2}-R_{1}^{2}}\left(\left.\frac{r^{2-\alpha}}{2-\alpha}\right|_{r=R_{1}} ^{R_{L}}\right) \\
& =\frac{2 P}{2-\alpha} \cdot \frac{R_{L}^{2-\alpha}-R_{1}^{2-\alpha}}{R_{L}^{2}-R_{1}^{2}},
\end{aligned}
$$

where $(a)$ follows from Lemma 3. Lastly, the mean of the sum of the interference from all interferers is simply the sum of their individual means, from which the result follows. 


\section{Proof of Lemma 5}

$$
\begin{aligned}
& \mathbb{E}\left[\mathcal{I}_{2} \mid R_{L}\right]=\mathbb{E}\left[\sum_{x \in \Phi \backslash \mathfrak{b}\left(o, R_{L}\right)} P\|x\|^{-\alpha}\right] \\
& =\lambda \int_{\mathfrak{b}^{c}\left(o, R_{L}\right)} P\|x\|^{-\alpha} \mathrm{d} x=P \lambda \int_{0}^{2 \pi} \int_{R_{L}}^{\infty} r^{-\alpha} r \mathrm{~d} r \mathrm{~d} \theta \\
& =2 P \pi \lambda \int_{R_{L}}^{\infty} r^{1-\alpha} \mathrm{d} r=\left.2 P \pi \lambda \cdot \frac{r^{2-\alpha}}{2-\alpha}\right|_{r=R_{L}} ^{\infty} \\
& =\frac{2 P \pi \lambda}{\alpha-2} R_{L}^{2-\alpha}
\end{aligned}
$$

for $\alpha>2$, and unbounded otherwise.

\section{E. Proof of Theorem 1}

Let $\sigma^{2}=0$, and evaluate at $\alpha=4$.

$$
\begin{aligned}
& \mathrm{P}_{\mathrm{L}}(L, \beta, \gamma, \lambda)=\mathbb{P}\left(\operatorname{SINR}_{L} \geq \gamma^{-1} \beta\right) \\
& =\mathbb{E}_{R_{L}}\left[\mathbb{E}_{R_{1}}\left[\mathbb{1}\left(\operatorname{SINR}_{L} \geq \gamma^{-1} \beta \mid R_{1}, R_{L}\right) \mid R_{L}\right]\right]
\end{aligned}
$$

The $\operatorname{SINR}_{L}$ term in the above expression is

$$
\begin{aligned}
\operatorname{SINR}_{L} & =\frac{P R_{L}^{-\alpha}}{P R_{1}^{-\alpha}+\mathbb{E}\left[\mathcal{I}_{1} \mid R_{1}, R_{L}\right]+\mathbb{E}\left[\mathcal{I}_{2} \mid R_{L}\right]} \\
& =\frac{P R_{L}^{-\alpha}}{P R_{1}^{-\alpha}+\frac{2 P(L-2)}{2-\alpha} \cdot \frac{R_{L}^{2-\alpha}-R_{1}^{2-\alpha}}{R_{L}^{2}-R_{1}^{2}}+\frac{2 P \pi \lambda}{\alpha-2} R_{L}^{2-\alpha}} \\
& =\frac{1}{\left(\frac{R_{L}}{R_{1}}\right)^{\alpha}+\frac{2(L-2)}{2-\alpha} \cdot \frac{\left(\frac{R_{L}}{R_{1}}\right)^{2}-\left(\frac{R_{L}}{R_{1}}\right)^{\alpha}}{\left(\frac{R_{L}}{R_{1}}\right)^{2}-1}+\frac{2 \pi \lambda}{\alpha-2} R_{L}^{2}} \\
& \stackrel{(a)}{=} \frac{1}{X^{4}+(L-2) X^{2}+\pi \lambda R_{L}^{2}},
\end{aligned}
$$

where (a) follows by defining $X=\frac{R_{L}}{R_{1}}$ and substituting $\alpha=4$. Using (14), we now simplify $\operatorname{SINR}_{L} \geq \gamma^{-1} \beta$ that will then used to derive $\mathrm{P}_{\mathrm{L}}(L, \beta, \gamma, \lambda)$ in (13). Defining $Y=X^{2}$ we have

$$
\begin{aligned}
& \operatorname{SINR}_{L} \geq \gamma^{-1} \beta \stackrel{(a)}{\Rightarrow} Y^{2}+(L-2) Y \leq \kappa^{-1} \\
& \Rightarrow\left(Y+\frac{(L-2)}{2}\right)^{2} \leq \kappa^{-1}+\frac{(L-2)^{2}}{4} \\
& \stackrel{(b)}{\Rightarrow} 0 \leq Y \leq \sqrt{\kappa^{-1}+\frac{(L-2)^{2}}{4}}-\frac{(L-2)}{2} \\
& \stackrel{(c)}{\Rightarrow} X^{2} \leq \sqrt{\kappa^{-1}+\frac{(L-2)^{2}}{4}}-\frac{(L-2)}{2} \\
& \stackrel{(d)}{\Rightarrow} 1 \leq X \leq \sqrt{\sqrt{\kappa^{-1}+\frac{(L-2)^{2}}{4}}-\frac{(L-2)}{2}} \\
& \Rightarrow \frac{R_{L}}{\sqrt{\sqrt{\kappa^{-1}+\frac{(L-2)^{2}}{4}}-\frac{(L-2)}{2}}} \leq R_{1} \leq R_{L}
\end{aligned}
$$

where $\kappa^{-1}=\gamma / \beta-\pi \lambda R_{L}^{2}$ in $(a),(b)$ follows from the fact that $Y \geq 1$, and $(c)$ from $Y=X^{2}$. Note that $(b)$ and $(c)$ require $\kappa^{-1} \geq-\frac{(L-2)^{2}}{4}$. Step $(d)$ follows from $X \geq 1$. The earlier condition on $\kappa^{-1}$ is replaced by a more strict condition $\kappa^{-1} \geq L-1$ in $(d)$. Substituting this back in (13) and doing some algebraic manipulations, we get

$$
\begin{aligned}
& \mathrm{P}_{\mathrm{L}}(L, \beta, \gamma, \lambda)=\mathbb{E}_{R_{L}}\left[\left(\frac{R_{L}^{2}-\frac{R_{L}^{2}}{\sqrt{\kappa^{-1}+\frac{(L-2)^{2}}{4}}-\frac{(L-2)}{2}}}{R_{L}^{2}}\right)^{L-1}\right] \\
& =\mathbb{E}_{R_{L}}\left[\left(1-\frac{1}{\sqrt{\gamma / \beta-\pi \lambda R_{L}^{2}+\frac{(L-2)^{2}}{4}}-\frac{(L-2)}{2}}\right)^{L-1}\right],
\end{aligned}
$$

from which the result follows by simply integrating across the density of $R_{L}$. Due to $\kappa^{-1} \geq L-1$ the integration limits are from 0 to $\sqrt{\frac{\gamma / \beta-(L-1)}{\pi \lambda}}$. This completes the proof.

\section{REFERENCES}

[1] D. J. Torrieri, "Statistical theory of passive location systems," IEEE Trans. Aerosp. Electron. Syst., vol. AES-20, no. 2, pp. 183-198, Mar. 1984.

[2] M. A. Spirito, "On the accuracy of cellular mobile station location estimation," in IEEE Trans. Veh. Technol., vol. 50, no. 3, 2001, pp. 674-685.

[3] J. H. Reed and K. J. Krizman, "An overview of the challenges and progress in meeting the E-911 requirement for location service," IEEE Commun. Mag., vol. 36, no. 4, pp. 30-37, 1998.

[4] Federal Communications Commission, "Wireless E911 location accuracy requirements," PS Docket No. 07-114, Jan. 2015.

[5] J. F. C. Kingman, Poisson Processes. Oxford University Press, 1993.

[6] M. Haenggi, Stochastic Geometry for Wireless Networks. New York: Cambridge University Press, 2013.

[7] Third Generation Partnership Project (3GPP), "R1-090053: Improving the hearability of LTE positioning service," Alcatel-Lucent, 3GPP TSG-RAN WG1 \#55bis, Ljubljana, Slovenia, Jan. 2009.

[8] — - "R1-091443: Evaluation parameters for positioning studies," Alcatel-Lucent, Ericsson, Motorola, Nokia, NSN, Nortel, Qualcomm Europe, 3GPP TSG-RAN WG1 \#56bis, Seoul, Korea, Mar. 2009.

[9] —-, "R1-091912: Discussions on UE positioning issues," Nortel, 3GPP TSG-RAN WG1 \#57, San Francisco, USA, May 2009.

[10] M. Haenggi, J. Andrews, F. Baccelli, O. Dousse, and M. Franceschetti, "Stochastic geometry and random graphs for the analysis and design of wireless networks," IEEE J. Sel. Areas Commun., vol. 27, no. 7, pp. 1029-1046, Sep. 2009.

[11] J. G. Andrews, F. Baccelli, and R. K. Ganti, "A tractable approach to coverage and rate in cellular networks," IEEE Trans. Commun., vol. 59, no. 11, pp. 3122-3134, Nov. 2011.

[12] H. S. Dhillon, R. K. Ganti, F. Baccelli, and J. G. Andrews, "Modeling and analysis of K-tier downlink heterogeneous cellular networks," IEEE J. Sel. Areas Commun., vol. 30, no. 3, pp. 550-560, Apr. 2012.

[13] B. Blaszczyszyn and M. K. Karray, "Quality of service in wireless cellular networks subject to log-normal shadowing," IEEE Trans. Commun., vol. 61, no. 2, pp. 781-791, Feb. 2013.

[14] H. S. Dhillon and J. G. Andrews, "Downlink rate distribution in heterogeneous cellular networks under generalized cell selection," IEEE Wireless Commun. Letters, vol. 3, no. 1, pp. 42 - 45, Feb. 2014.

[15] B. Blaszczyszyn, M. K. Karray, and H. P. Keeler, "Using Poisson processes to model lattice cellular networks," in 2013 Proc. IEEE INFOCOM, Apr. 2013, pp. 773-781.

[16] H. P. Keeler, N. Ross, and A. Xia, "When do wireless network signals appear Poisson?" arXiv:1411.3757 [math.PR].

[17] M. Haenggi, "On distances in uniformly random networks," IEEE Trans. Inf. Theory, vol. 51, no. 10, pp. 3584-3586, Oct. 2005.

[18] J. Schloemann, H. S. Dhillon, and R. M. Buehrer, "Towards a tractable analysis of localization fundamentals in cellular networks," arXiv:1502.06899 [cs.IT].

[19] H. Urkowitz, Signal Theory and Random Processes. Norwood, MA: Artech House, 1983.

[20] H. P. Keeler, B. Blaszczyszyn, and M. K. Karray, "SINR-based k-coverage probability in cellular networks with arbitrary shadowing," in IEEE Int Symp. Inf. Theory, Jul. 2013, pp. 1167-1171. 